\title{
Uma Abordagem Baseada em Algoritmo Genético para a Suavização de Caminhos para Espuma Probabilística
}

\author{
Luís B. P. Nascimento* Vitor G. Santos* Diego S. Pereira** \\ Dennis Barrios-Aranibar ${ }^{* * *}$ Raquel E. Patiño-Escarcina ${ }^{* * *}$ \\ Daniel S. Morais* Victor C. A. Pimentel ${ }^{* *}$ Pablo J. Alsina* \\ * Departamento de Engenharia de Computação e Automação, \\ Universidade Federal do Rio Grande do Norte, RN, Brasil (e-mail: \\ \{lbruno,vitorgaboardi\}@ufrn.edu.br,pablo@dca.ufrn.br). \\ ** Instituto Federal do Rio Grande do Norte, RN, Brasil \\ *** Departamento de Ingeniería Eléctrica y Electrónica, Universidad \\ Católica San Pablo, Peru (e-mail: dbarrios@ucsp.edu.pe)
}

\begin{abstract}
Probabilistic Foam Method is an interesting robotic path planning technique that creates a free-obstacle path and ensures a safety maneuverability region. However, this algorithm may generate paths with many sharp or angular turns. In such a case, a smoothing strategy is desirable because it improves the path which the robot will follow. In this paper, the smoothing process is considered using Genetic Algorithm optimization, a powerful metaheuristic inspired in biological processes and theory of evolution. Simulations prove that it was possible to smooth paths generated by the Probabilistic Foam Method maintaining the safety constraints.

Resumo: O método da Espuma Probabilística é uma interessante técnica de planejamento de caminhos para robôs, pois além de gerar caminhos livre de obstáculos, ainda garante uma região para manobrabilidade segurança. Entretanto, são caminhos que necessitam de um processo de suavização. A estratégia de suavização a ser aplicada nesses caminhos devem respeitar as restrições de segurança impostas a eles. Dessa forma, o processo de suavização foi modelado como um problema de otimização para o Algoritmo Genético, uma poderosa meta-heurística inspirada em processos biológicos e de evolução das espécies. Por meio de simulações foi possível suavizar caminhos gerados a partir do método da espuma probabilística, respeitando suas restrições de segurança.
\end{abstract}

Keywords: Path Smoothing; Path Planning; Autonomous Robot; Genetic Algorithm; Probabilistic Foam.

Palavras-chaves: Suavização de Caminhos; Planejamento de Caminhos; Robótica Autônoma; Algoritmo Genético; Espuma Probabilística.

\section{INTRODUÇÃO}

O planejamento de caminho é um dos principais problemas da robótica autônoma, pois visa calcular um caminho para que o robô se mover de um ponto (configuração inicial $q_{\text {inicial }}$ ) a outro (configuração final $q_{\text {final }}$ ), sem colidir com possíveis obstáculos no ambiente (Svestka and Overmars, 1998). Existem diversos métodos de planejamento como a Árvore Aleatória de Exploração Rápida (RapidlyExploring Ramdom Tree - RRT) (Lavalle, 1998; Lavalle et al., 2000) e o Mapa de Rotas Probabilístico (Probabilistic Roadmaps) (Kavraki et al., 1996) que são os principais métodos de planejamento da atualidade.

Silveira e Alsina (2016) apresentaram um método de planejamento de caminho chamado Espuma Probabilística (Probabilistic Foam Method - PFM) onde, além de retornar

\footnotetext{
* O presente trabalho foi realizado com apoio da Coordenação de Aperfeiçoamento de Pessoal de Nível Superior - Brasil (CAPES) Código de Financiamento 001.
}

um caminho livre de obstáculos, restrições de segurança são impostas por meio de regiões no espaço livre que garantem uma manobrabilidade segura para o robô. Essa é uma estratégia interessante, principalmente para sistemas robóticos que necessitam de um maior grau de segurança nos caminhos, como mostra em Nascimento et al. (2018).

Todos esses métodos retornam caminhos compostos por um conjunto de pontos (configurações) ligados por segmentos de reta. Esses caminhos gerados pelos planejamentos são geralmente grosseiros, podendo apresentar curvas fechadas, o que dificulta a realização de manobras dependendo da cinemática do robô (Ravankar et al., 2018). A Figura 1 ilustra a diferença entre um caminho planejado e um caminho ideal.

Existem algumas estratégias que podem ser aplicadas em caminhos mais grosseiros a fim de suavizá-los. As estratégias mais comuns são baseadas em Interpolação (Waring, 1779), como curvas de Bézier (Zhou et al., 2011), Splines (Ahmed and Deb, 2011), entre outras. Uma estratégia inte- 


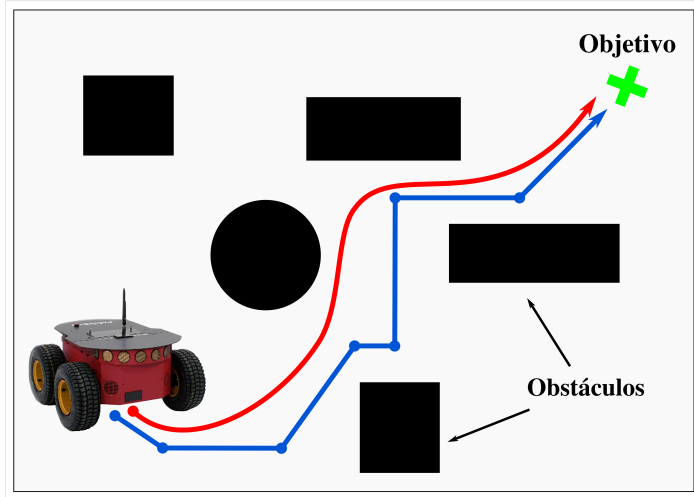

Figura 1. A linha azul representa um caminho composto por segmentos de reta com curvas fechadas. O caminho em vermelho é mais suave para o mesmo objetivo.

ressante pode ser o uso de técnicas de otimização, uma vez que suavizar caminho pode ser considerado um problema de otimização. Além disso, Ravankar et al. (2018) mostra que técnicas de otimização são ideais para problemas de suavização com muitas restrições. Uma poderosa e popular técnica de otimização e que é capaz de resolver diversos problemas é o Algoritmo Genético (Genetic Algorithm GA), uma meta-heurística inspirada em conceitos como evolução das espécies.

Motivado pela necessidade de suavizar os caminhos gerados pelo método PFM, respeitando suas restrições de segurança, esse trabalho apresenta a aplicação da técnica de otimização Algoritmo Genético na suavização de caminhos extraídos por meio do método de planejamento Espuma Probabilística.

O resto do trabalho está organizado como segue: A Seção 2 apresenta o Algoritmo Genético. Na seção 3 é apresentado o método da Espuma Probabilística. Na Seção 4 o problema da suavização do caminho obtido pelo PFM é modelado como um problema de otimização. Na Seção seguinte alguns resultados são apresentados e finalmente, as conclusões são apresentadas na Seção 6.

\section{ALGORITMO GENÉTICO}

O Algoritmo Genético (Genetic Algorithm - GA) é uma meta-heurística inspirada em conceitos oriundos da teoria da evolução das espécies. O GA trata do princípio da seleção natural dos seres vivos e sobrevivência do mais aptos, estabelecida por Charles Darwin, elementos da genética, como o cruzamento de cromossomos, proposta por Gregor Mendel e da teoria da mutação, apresentada por Hugo de Vries. O GA pode atuar na resolução de diversos problemas englobando os de busca e otimização. Essa meta-heurística foi introduzida por Holland (1975) e foi popularizada por Goldberg (1989).

Nesse algoritmo, um conjunto de possíveis soluções (população inicial) são geradas aleatoriamente dentro de um espaço de busca. Todas as possíveis soluções (indivíduos) da população são avaliadas por meio de uma função objetivo (função de avaliação ou fitness) e um conjunto de indivíduos melhores avaliados (mais aptos) são selecionados e, assim, ocorrem as principais operações do GA para geração de filhos: Cruzamento, onde ocorre a troca ou mistura do material genético dos pais, e a Mutação, onde ocasionalmente pode ocorrer uma alteração no material genético, modificando o indivíduo. Por meio do Elitismo, indivíduos mais aptos podem ser mantidos na população e os demais são substituídos pelos novos filhos gerados, finalizando assim uma geração. Um pseudocódigo com as etapas do Algoritmo Genético está apresentado no Algoritmo 1.

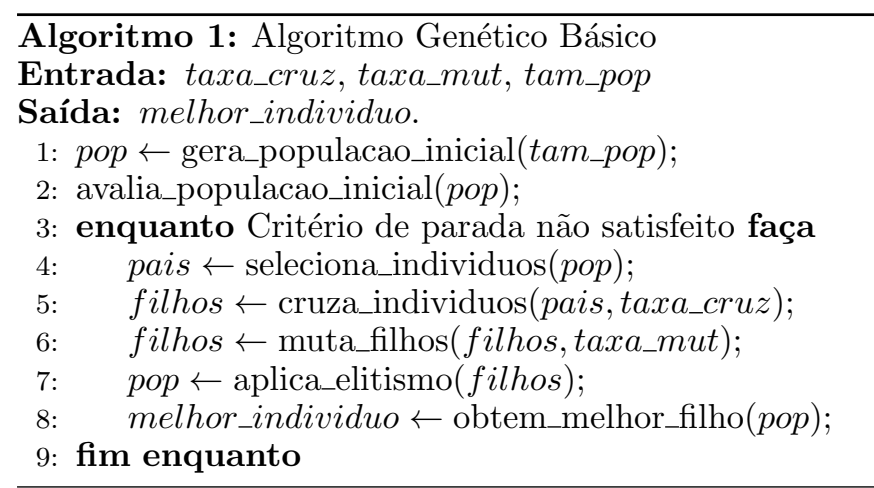

No GA existem alguns parâmetros importantes para o processo de convergência. O parâmetro tam_pop é o tamanho da população gerada. A taxa de cruzamento taxa_cruz representa a probabilidade de um cruzamento ocorrer e geralmente é um valor alto. A taxa de mutação taxa_mut representa a probabilidade de uma mutação ocorrer, e geralmente é um valor pequeno usado para garantir a variabilidade genética.

\section{O MÉTODO DA ESPUMA PROBABILÍSTICA}

Seja $C$ o espaço $n$-dimensional de todas as possíveis configurações $q$ que um robô pode assumir. Define-se $C$ o espaço de configurações, $C_{o}$ o subconjunto de $C$ contendo obstáculos e $C_{f}$ o subconjunto contendo o espaço livre de obstáculos. Conforme Silveira and Alsina (2016), uma bolha $b$ de raio $r$ centrada na configuração $q_{c} \in C_{f}$ é uma $n$ - bola definida como:

$$
b=b\left(q_{c}, r\right)=\left\{q / d\left(q, q_{c}\right)<r\right\}
$$

sendo $d\left(q, q_{c}\right)$ a métrica utilizada em $C$ e $r$ é a distância mínima entre $q_{c}$ e $C_{o}(2)$.

$$
r=\min _{q^{\prime} \in C_{o}} d\left(q^{\prime}, q_{c}\right)
$$

Dessa forma, pressupõe-se que uma bolha centrada em $q_{c}$ pode expandir-se partindo do seu centro até o obstáculo mais próximo.

A espuma probabilística $E \subset C_{f}$ é definida como uma coleção de bolhas sobrepostas, isto é, $E=\cup b$. Um conjunto de bolhas (bolhas filhas) pode ser expandido na superfície de outra bolha (bolha mãe), tal que, uma bolha filha é centrada na superfície de uma bolha mãe, formando obrigatoriamente uma região de interseção entre as bolhas chamada "escotilha". Internamente a essa região de interseção, existe uma região de dimensão $n-1$ chamada "portal", a qual garante uma navegabilidade segura entre as bolhas. O portal é ilustrado na Figura 2.

\subsection{A propagação da espuma}

A propagação da espuma probabilística através de $C_{f}$ ocorre por meio da expansão de bolhas filhas na superfície 


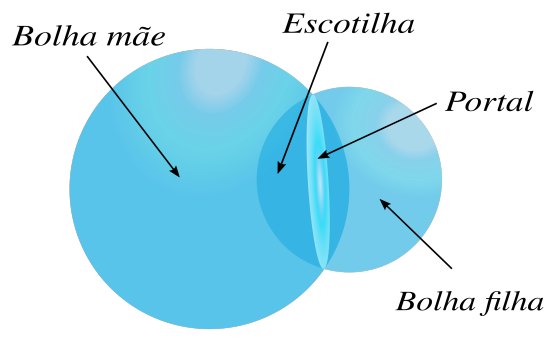

Figura 2. Portal formado entre duas bolhas mãe e filha.

livre de bolhas mães. Considerando que a superfície de uma bolha é uma $(n-1)$-esfera em $C_{f}$, o número $N$ máximo possível de bolhas filhas que uma bolha mãe pode gerar em sua superfície é:

$$
N=K\left(\left\lfloor\frac{r}{r_{\min }}\right\rfloor\right)^{n-1}
$$

O parâmetro $r_{\min }$ é uma constante que indica o menor raio admissível para uma bolha. A constante $K$ define o número máximo de bolhas filhas que as bolhas com raio $r_{\text {min }}$ podem gerar.

Um total de $N$ configurações são amostradas sobre a superfície da bolha mãe, entretanto, é verificado se o ponto está localizado numa região já coberta por outra bolha (exceto a bolha mãe). Somente se o ponto estiver numa região descoberta, a bolha é expandida, porém, a bolha só será aceita se respeitar a condição de raio mínimo.

Todas as bolhas aceitas são armazenadas em uma árvore de busca, sendo que, cada nova bolha filha é adicionada à fila $F$. A propagação da espuma é um processo contínuo de seleção de bolhas em $F$ e expansão de bolhas filhas, até que uma bolha englobe a configuração $q_{\text {final }}$, e assim, o processo de busca finaliza. Uma ilustração da espuma probabilística formada é mostrada na Figura 3a.

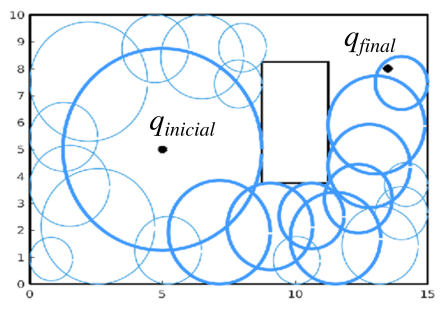

(a)

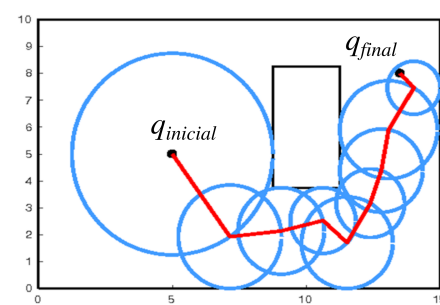

(b)
Figura 3. (a) Ilustração da espuma probabilística formada.

(b) Rosário extraído da espuma probabilística.

Por meio de uma busca partindo da bolha que engloba a configuração $q_{\text {final }}$, seguindo o grau de parentesco entre as bolhas, é possível encontrar o rosário $\mathcal{R}$, uma região definida como uma sequência de bolhas sobrepostas, partindo da bolha final até a bolha inicial.

$$
\mathcal{R}=\left\{b_{1}, b_{2}, \ldots, b_{k-1}, b_{k} \mid b_{1}=b_{\text {final }} \text { e } b_{k}=b_{\text {inicial }}\right\}
$$

A partir do rosário $\mathcal{R}$ é possível extrair um caminho factível $\sigma$ entre a configuração inicial até a configuração final.
Na Figura 3b é mostrado um caminho (linha vermelha) obtido por meio de segmentos de reta ligados pelos centros das bolhas do rosário.

\section{MODELAGEM DO PROBLEMA}

Para a aplicação do GA para a suavização dos caminhos gerados pelo PFM, é necessário modelar esse problema como um problema de otimização por GA. Como já foi comentado, a navegação entre duas bolhas deve ocorrer por meio da região de interseção, passando consequentemente, pelo portal. Dessa maneira, para garantir essa restrição, o GA deve gerar um caminho, tal que, os pontos estejam limitados pela escotilha. Assim, o ajuste poderá ocorrer e, com a restrição respeitada, o caminho continuará seguro.

\subsection{Codificação do indivíduo}

Como comentado anteriormente, a solução do problema de suavização será um caminho composto por segmentos de reta ligados por pontos que passam obrigatoriamente pela escotilha das bolhas do rosário. Sendo assim, considere a Figura 4 que ilustra um rosário extraído de uma espuma probabilística. É importante observar que ao respeitar as restrições representadas pelas regiões em azul na figura, o caminho suavizado dentro desse rosário nunca poderia ser uma reta, que representaria a situação ideal para este caso.

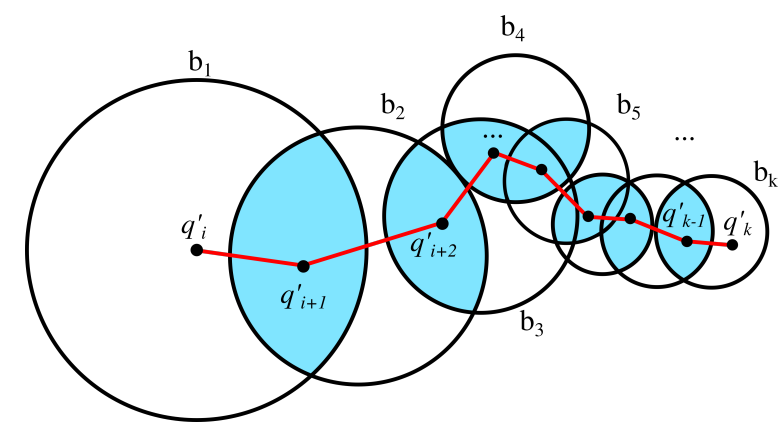

Figura 4. Rosário com um caminho representando uma possível solução do problema.

Durante a criação da população inicial o GA deve gerar caminhos, onde $q^{\prime}$ representa configurações de cada uma das escotilhas. Isso indica que o algoritmo vai tentar suavizar o caminho sem modificar a estrutura do rosário. Uma outra informação importante é que as configurações inicial e final não devem se alterar, mantendo fixas as extremidades do caminho. Dessa forma, o vetor que representa os indivíduos para o GA é definido por:

$$
\sigma=\left\{q_{1}^{\prime}, q_{2}^{\prime}, \ldots, q_{k-2}^{\prime}, q_{k-1}^{\prime}\right\}
$$

sendo $k$ o número de bolhas no rosário.

\subsection{A Função Objetivo}

Para avaliar cada possível solução para o problema, foi considerado neste trabalho o valor dos ângulos formados entre cada par de segmentos de reta no caminho $\sigma$. A Figura 5 ilustra o caminho extraído pelo rosário mostrado na Figura 4. 


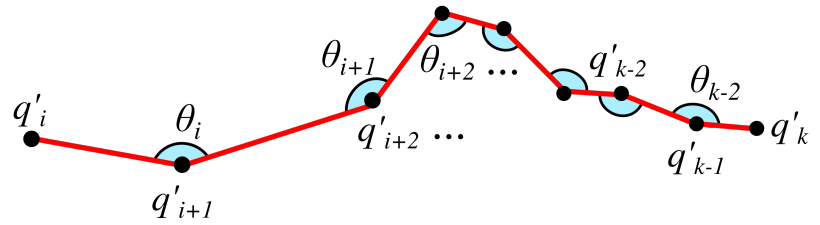

Figura 5. Ângulos formados pelos segmentos de reta no caminho.

Os ângulos gerados a partir dos pares de segmentos de reta dos caminhos, como mostrado na Figura 5, podem ser calculados como segue:

$$
\theta_{i}=\cos ^{-1} \frac{\left(q_{i}^{\prime}-q_{i+1}^{\prime}\right) \cdot\left(q_{i+2}^{\prime}-q_{i+1}^{\prime}\right)}{\left|q_{i}^{\prime}-q_{i+1}^{\prime}\right|\left|q_{i+2}^{\prime}-q_{i+1}^{\prime}\right|}
$$

A partir dos valores dos ângulos é possível calcular a avaliação do caminho por meio da função $f(\sigma)$, melhor definida em Eq. (7):

$$
\begin{aligned}
& \min f(\sigma)=\frac{\sum_{i=1}^{k-2}\left(\pi-\theta_{i}\right)}{k-2} \\
& \text { Sujeito à: } \\
& q_{i}^{\prime} \in\left(b_{i} \cap b_{i+1}\right)
\end{aligned}
$$

onde $b_{i} \cap b_{i+1}$ é a escotilha, região de interseção entre duas bolhas de grau de parentesco direto e centradas nas configurações $q_{i}$ e $q_{i+1}$, respectivamente. A minimização da função $f(\sigma)$ indica a abertura dos ângulos $\theta$ formados no caminho, tornando assim o caminho mais suave.

\subsection{Estratégias utilizadas na implementação do GA}

Na implementação desse Algoritmo Genético foram utilizados operadores para valores reais. O processo de seleção foi realizado através de sorteio por Roleta, atribuindo maior probabilidade aos indivíduos melhores avaliados, isto é, com menor valor de $f(\sigma)$.

A reprodução dos indivíduos ocorreu por meio de um Cruzamento Heurístico (Wright, 1991; Herrera and Lozano, 1996). Nessa estratégia, um novo indivíduo $\sigma_{f}$ foi gerado por meio de uma combinação entre dois indivíduos $\sigma_{p}^{1} \mathrm{e}$ $\sigma_{p}^{2}$. Considerando que $f\left(\sigma_{p}^{1}\right) \leqslant f\left(\sigma_{p}^{2}\right)$, o indivíduo $\sigma_{f}$ foi gerado como:

$$
\sigma_{f}=\sigma_{p}^{1}+\beta\left(\sigma_{p}^{1}-\sigma_{p}^{2}\right)
$$

sendo $\beta$ um valor randômico uniforme, tal que $\beta \in[0,1]$.

$\mathrm{Na}$ operação de Mutação, os alelos de um indivíduo (as configurações $\left\{q_{i}^{\prime}, q_{i+1}^{\prime}, \ldots, q_{k-1}^{\prime}\right\}$ (conforme (5)) foram modificados por meio de um ajuste sutil. Sendo $\alpha$ um valor aleatório uniforme no intervalo $[a, b]$, isto é, $\alpha \in[a, b], q_{i}^{\prime}$ foi ajustado por $\alpha q_{i}^{\prime}$. Além disso, após o ponto ter sido modificado, é verificado se ele ainda pertence à escotilha. Se não, ele retorna o valor anterior. Por fim, o elitismo foi implementado através da substituição do indivíduo com pior avaliação pelo melhor indivíduo da geração anterior.

\section{RESULTADOS}

Para validar a metodologia apresentada, foram realizados planejamentos de caminhos por meio do PFM para dois mapas diferentes, mostrados na Figura 6.

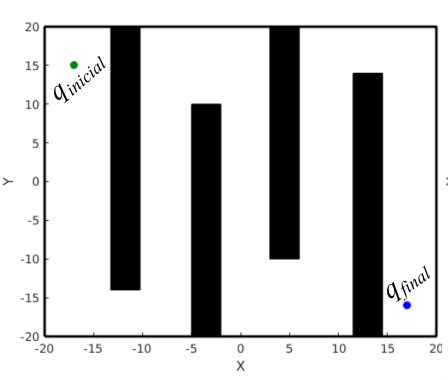

(a)

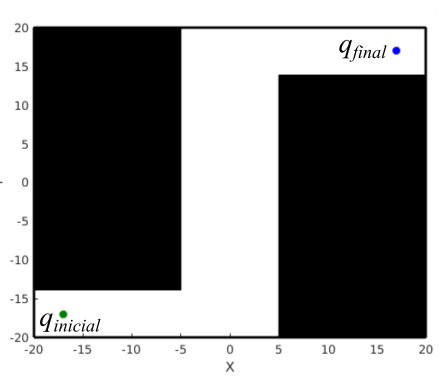

(b)
Figura 6. Mapa 1 (a) e Mapa 2 (b).

A partir da simulação realizada com o PFM, foi possível obter um rosário para cada mapa e consequentemente, seus respectivos caminhos, sendo submetidos ao processo de suavização por GA, de forma a garantir a restrição de segurança do rosário. A Tabela 1 apresenta os parâmetros utilizados na implementação do Algoritmo Genético. Esses parâmetros foram escolhidos de maneira experimental.

Tabela 1. Parâmetros do Algoritmo Genético.

\begin{tabular}{cccc} 
Tam. população & Taxa cruzamento & Taxa mutação & $\alpha$ \\
\hline 150 & 0,4 & 0,01 & {$[0,9,1,1]$} \\
\hline
\end{tabular}

Um detalhe importante a ser observado é que a taxa de cruzamento precisou assumir um valor bem pequeno, o que não é usual na maioria das aplicações. Essa escolha foi necessária por conta da sensibilidade do caminho à operação de cruzamento, o que evitou convergências precoces. Na operação de mutação, além de assumir um valor pequeno na taxa de mutação, a operação em si funcionou como um ajuste fino com $\alpha \in[0,9,1,1]$, pois alterações mais groseiras estavam comprometendo a convergência do GA.

O critério de parada do GA foi por meio da variabilidade dos indivíduos da população. Quando o algoritmo convergiu de tal maneira que não houve variação aparente no valor médio da fitness da população, o algoritmo parou. $\mathrm{O}$ resultado da suavização do caminho obtido para o Mapa 1 é apresentado nas Figuras 7, 8 e 9.

O caminho mostrado na Figura 7 apresenta o rosário obtido com o planejamento e o caminho extraído que possui fitness de $f(\sigma)=0,7195$. O caminho resultante após a suavização é mostrado na Figura 8.

O caminho apresentado na Figura 8 corresponde ao melhor indivíduo da população após a execução do GA. A fitness desse indivíduo foi de aproximadamente $f(\sigma)=0,3269$. A curva de convergência referente à execução do GA para esse resultado é mostrada na Figura 9.

A curva apresentada na Figura 9 representa a média da fitness de todos os indivíduos na população e mostra que apenas com 25 gerações o método já iniciou o processo de estagnação, convergindo para um determinado indivíduo. 


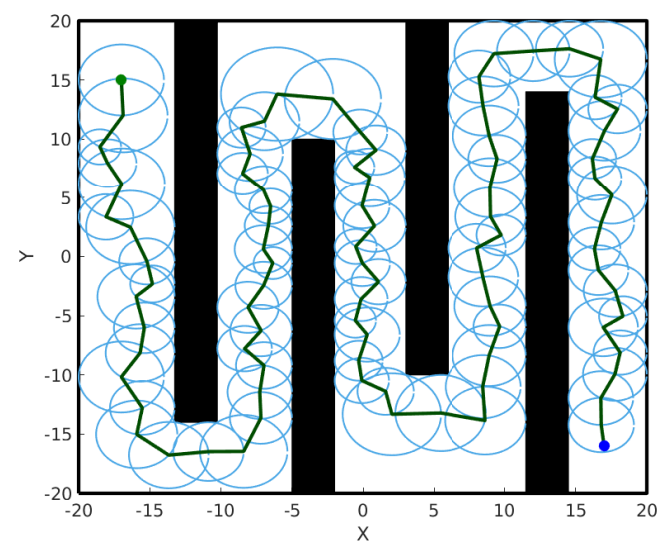

Figura 7. Rosário e caminho extraídos da Espuma Probabilística para o Mapa 1.

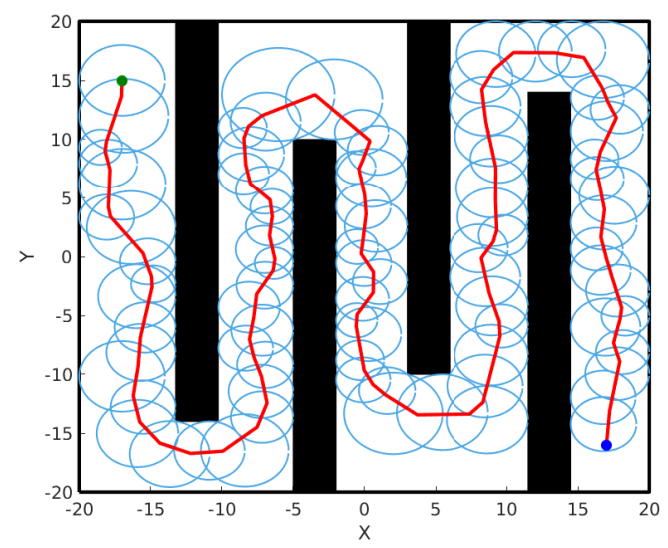

Figura 8. Caminho do Mapa 1 suavizado pelo GA.

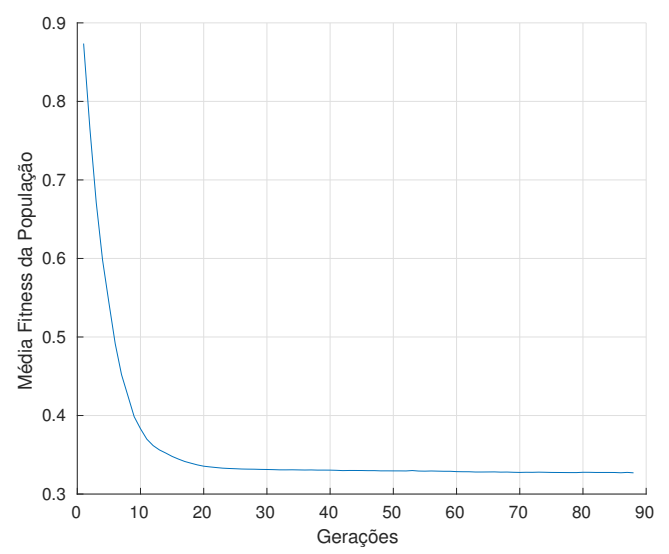

Figura 9. Curva de convergência para o Mapa 1.

A população apresentou fitness média de $f(\sigma)=0,3270$ e com desvio padrão $s t d=0,0003$.

É possível perceber na simulação que a estratégia foi capaz de tornar o caminho mais suave quando comparado ao resultado obtido com o planejamento, entretanto, o Algoritmo Genético não convergiu de maneira ótima. Isso ocorreu principalmente por conta da própria restrição imposta pelo rosário. As bolhas que constituíram o rosário possuem tamanhos irregulares e estão posicionadas de forma a dificultar o processo de suavização.

Observe agora nas Figuras 10 e 11 os resultados da suavização para o Mapa 2.

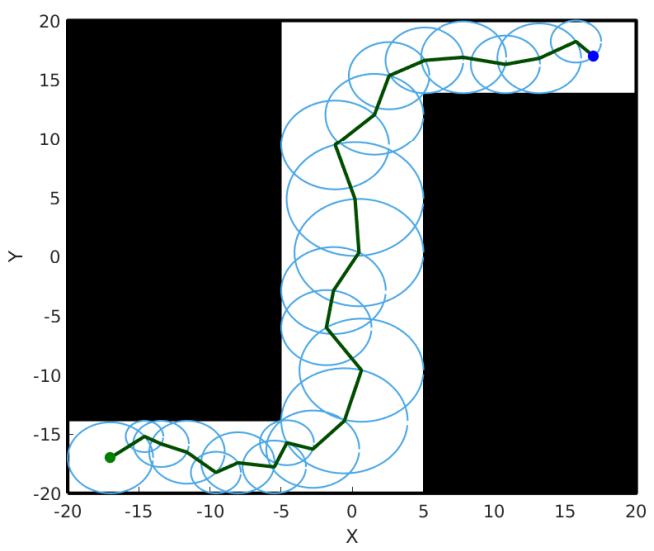

Figura 10. Rosário e caminho extraídos da Espuma Probabilística para o Mapa 2.

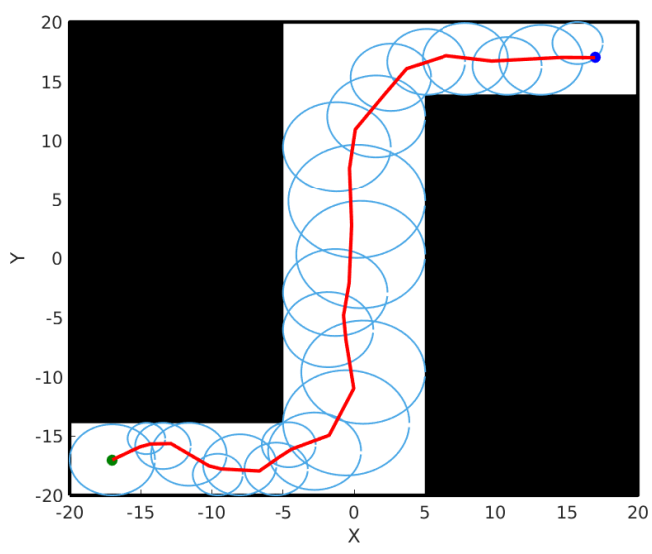

Figura 11. Caminho do Mapa 2 suavizado pelo GA.

O caminho extraído do rosário na Figura 10 foi avaliado com $f(\sigma)=0,7103$. Com a suavização do caminho com GA, mostrado na Figura 11, a fitness foi de aproximadamente $=f(\sigma)=0,2993$. O GA fez com que a população convergisse e atingisse o valor médio da avaliação de aproximadamente $f(\sigma)=0,2993$, com desvio padrão $s t d=0,0002$. De maneira similar ao ocorrido com o Mapa 1, o GA tornou o caminho mais suave que o caminho gerado pelo PFM, porém é possível perceber que o rosário gerado desfavorece o processo de suavização devido ao posicionamento e tamanho irregular das bolhas. Para ilustrar esse problema, observe a Figura 12.

Um rosário foi gerado manualmente dentro do Mapa 2 contendo bolhas regulares de acordo com a largura do corredor, e posicionadas de maneira organizada, de modo a proporcionar uma melhor cobertura do corredor. Ligando os centros dessas bolhas por meio de segmentos de reta, foi possível obter um caminho bem mais suave em comparação ao obtido com o GA, apresentando fitness de $f(\sigma)=$ 0,1963 . Além disso, o caminho obtido está mais afastado da região de obstáculos e, consequentemente, mais seguro. 


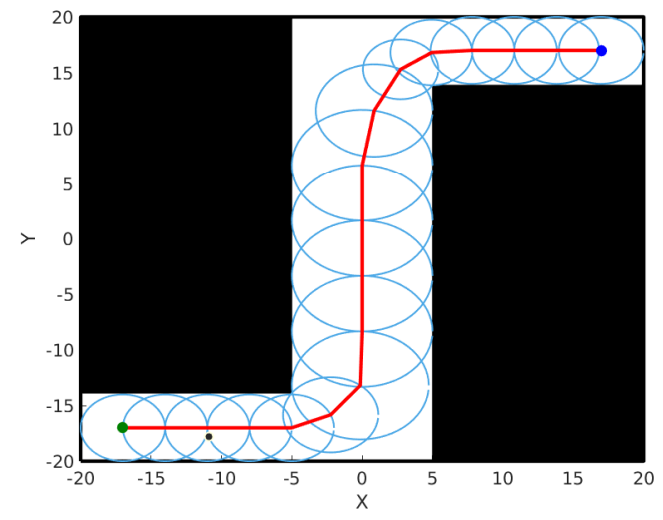

Figura 12. Rosário gerado manualmente para ilustrar um resultado ideal para o Mapa 2.

\section{CONCLUSÕES}

Este artigo apresentou a aplicação do Algoritmo Genético no processo de suavização de caminhos robóticos com restrições. O caminho objeto de estudo foi calculado pelo método da Espuma Probabilística que gerou uma estrutura chamada Rosário, formada por um conjunto de bolhas que garantiram uma região de segurança para o caminho. O problema da suavização foi modelado como um problema de otimização e testado com o GA para verificar a eficácia da resolução do problema por meio de uma metaheurística.

Foi possível perceber que o GA foi capaz de suavizar os caminhos irregulares gerados pelo PFM respeitando a restrição de segurança gerada por meio da interseção entre duas bolhas do rosário. A escolha correta dos parâmetros e dos operadores do GA foi de grande importância para a convergência da população, pois o caminho se mostrou muito sensível às operações de cruzamento e mutação, assim, os ajustes no caminho tiveram que ser sutis.

Entretanto, foi observado que o GA não foi capaz de gerar uma suavização ótima nos caminhos. Isso provavelmente ocorreu pela natureza do próprio rosário, pois as restrições impõem que o caminho passe por todas as bolhas, e se houverem bolhas de tamanhos muito variados e estiverem posicionadas de maneira desfavorável, o caminho não apresentará uma forma muito suave. Outra possibilidade que pode ter influenciado na convergência foi que os parâmetros dos operadores assumiram valores estáticos e com o avançar das gerações, os ajustes vão tornando-se mais grosseiros, fazendo com que o método não melhore os resultados.

Dessa maneira, sugere-se como trabalhos futuros, a utilização de operadores adaptativos para ajustar o caminho da melhor maneira conforme as gerações. Sugere-se também a utilização de estratégias que possam ajustar não só o caminho, mas também o rosário, garantindo a segurança no caminho e uma melhor suavidade. Finalmente, é interessante a aplicação de outras técnicas de otimização nesse problema para verificar a possibilidade de melhora nos resultados apresentados.

\section{REFERÊNCIAS}

Ahmed, F. and Deb, K. (2011). Multi-objective path planning using spline representation. In 2011 IEEE International Conference on Robotics and Biomimetics, 1047-1052. doi:10.1109/ROBIO.2011.6181426.

Goldberg, D.E. (1989). Genetic Algorithms in Search, Optimization and Machine Learning. Addison-Wesley Longman Publishing Co., Inc., Boston, MA, USA, 1st edition.

Herrera, F. and Lozano, M. (1996). Heuristic crossovers for real-coded genetic algorithms based on fuzzy connectives. In International Conference on Parallel Problem Solving from Nature, 336-345. Springer.

Holland, J.H. (1975). Adaptation in Natural and Artificial Systems. University of Michigan Press, Ann Arbor, MI. Second edition, 1992.

Kavraki, L.E., Svestka, P., Latombe, J.., and Overmars, M.H. (1996). Probabilistic roadmaps for path planning in high-dimensional configuration spaces. IEEE Transactions on Robotics and Automation, 12(4), 566-580. doi:10.1109/70.508439.

Lavalle, S.M. (1998). Rapidly-exploring random trees: A new tool for path planning.

Lavalle, S.M., Kuffner, J.J., and Jr. (2000). RapidlyExploring Random Trees: Progress and Prospects. In Algorithmic and Computational Robotics: New Directions, 293-308.

Nascimento, L.B.P., Pereira, D.S., Sanca, A.S., Eugenio, K.J.S., Fernandes, D.H.S., Alsina, P.J., Araujo, M.V., and Silva, M.R. (2018). Safe path planning based on probabilistic foam for a lower limb active orthosis to overcoming an obstacle. In 2018 Latin American Robotic Symposium, 2018 Brazilian Symposium on Robotics (SBR) and 2018 Workshop on Robotics in Education (WRE), 413-419. doi:10.1109/LARS/SBR/WRE.2018. 00080.

Ravankar, A., Ravankar, A., Kobayashi, Y., Hoshino, Y., and Peng, C.C. (2018). Path smoothing techniques in robot navigation: State-of-the-art, current and future challenges. Sensors, 18(9), 3170.

Silveira, Y.S. and Alsina, P.J. (2016). A New Robot Path Planning Method Based on Probabilistic Foam. In 2016 XIII Latin American Robotics Symposium and IV Brazilian Robotics Symposium (LARS/SBR), 217-222. doi:10.1109/LARS-SBR.2016.43.

Svestka, P. and Overmars, M.H. (1998). Probabilistic Path Planning. In M. Thoma (ed.), Robot Motion Planning and Control, volume 229 of Lecture Notes in Control and Information Sciences, 255-304. Springer Berlin Heidelberg, Berlin, Heidelberg. doi:10.1007/ BFb0036069.

Waring, E. (1779). Vii. problems concerning interpolations. Philosophical transactions of the royal society of London, 69, 59-67.

Wright, A.H. (1991). Genetic algorithms for real parameter optimization. In Foundations of genetic algorithms, volume 1, 205-218. Elsevier.

Zhou, F., Song, B., and Tian, G. (2011). B \'\{e\} zier curve based smooth path planning for mobile robot. Journal of Information \& Computational Science, 8(12), 24412450 . 